\title{
Effet d'un dispositif biomécanique sur la récupération des sols salés et la culture du riz dans le bassin fluvio-marin du Sine-Saloum à Ndoff (Sénégal).
}

\author{
Cheikh Ahmeth Tidiane SEYE* (1), Elhadji FAYE (2), Abou THIAM (1), François MATTY (1) et \\ Bienvenu SAMBOU (2) \\ 1 Laboratoire de Science du Sol, Institut des Sciences de l'Environnement (ISE), Faculté des Sciences et \\ Techniques, Université Cheikh Anta Diop de Dakar (UCAD), BP 5005, Dakar-Fann, Sénégal. \\ 2 Institut Supérieur de Formation Agricole Rurale, Université de Thiès (UT), BP 967, Thiès. \\ *Correspondance, e-mail : cheikhseye88@yahoo.fr.
}

Original submitted in on $6^{\text {th }}$ September 2018. Published online at www.m.elewa.org on $31^{\text {st }}$ October 2018 https://dx.doi.org/10.4314/jab.v130i1.4

\section{RESUME}

Objectif : La salinisation est l'un des principaux facteurs de dégradation chimique des sols au Sénégal. Les terres salées de la zone du Sine Saloum augmentent constamment et représentent environ 33\% des terres émergées, selon Chauvin (2013). Pour faire face à cette contrainte, la présente étude expose les effets d'un dispositif biomécanique sur les sols salés et la culture du riz à Ndoff dans le bassin fluviomarin du SineSaloum.

Méthodologie et résultats : Le dispositif expérimental est en split plot avec 3 répétitions. Le facteur principal est l'espèce de reboisement (fixation des diguettes) avec 3 variantes (Gossypium hirsutum L. (GH), Andropogon gayanus. Kunt. (AG) et un témoin). Le facteur secondaire est la variété de riz avec 3 variantes («Rock 5 », "WAR 77 » et « Diayi »). Le nombre de répétitions est de 3. La caractérisation des sols du site expérimental a permis d'identifier un seul type de sol qui est hydromorphe minéral, salé, sulfaté acide, sur matériau sableux alluvio-colluvionnaire. Une baisse significative de la conductivité électrique (CE) et une augmentation du pH montrent que le dispositif biomécanique mis en place a fortement amélioré les caractéristiques physico-chimiques du sol. La variété locale de riz Diayi, en présence d'une plantation de $G$. hirsutum $\mathrm{L}$. $(\mathrm{GH})$ sur les diguettes, a obtenu la moyenne la plus importante pour le nombre total de talles productives, le nombre de plants avec épis et la longueur des épis. Le rendement grain de la première campagne a été nul à cause d'un déficit pluviométrique mais celui de la biomasse a été encourageant $\left(10,96\right.$ t.ha-1 $\left.^{-1}\right)$.

Conclusion et application des résultats : Ainsi, l'aménagement biomécanique des sols, accompagné d'une bonne fertilisation organo-minérale et de bonnes pratiques culturales et d'un choix de variétés adaptées est un procédé efficace pour la récupération et valorisation des terres salées dans la vallée de Ndoff. Toutefois, cette expérience mériterait d'être menée avec d'autres variétés de riz améliorées à cycle court.

Mots clés : Fatick, Ndoff, salinité, acidité, biomécanique, récupération, riz 


\section{ABSTRACT}

Objective: The main factors of chemical soil degradation in Senegal is salinization (Sadio 1991). The saline lands of the Sine Saloum area are constantly increasing and represent about $33 \%$ of the land surface, according to Chauvin (2013). To address this constraint, the present study exposes the effects of biomechanical design on saline soils and rice cultivation in the Sine-Saloum fluviomarine basin.

Methodology and results: The experimental design is a split plot with the species factor of small bunds fixation with three variants (main factor) and the variety factor with three variants (secondary factor). The three rice varieties used are: Diayi (local variety), the War 77 and Rock5 (new varieties). The three plant species for fixing the bunds are: Gossypium hirsutum L. (GH), Andropogon gayanus. Kunt (AG) and Vetiveria zizanioides L. (VZ). The number of repetitions is 3 . The results of the experimental site soil characterization revealed that the ground on which the test was conducted is a mineral, salty, acid, sulfated hydro-morph soil, on sandy alluvial-colluvial material. A significant decrease in the electrical conductivity (EC) and an increase in $\mathrm{pH}$ show that the established biomechanical device has greatly improved the physical and chemical soil characteristics. Local rice variety (Diayi) in the presence of a plantation of $G$. hirsutum $\mathrm{L}$. $(\mathrm{GH})$ on the bunds, received the largest average for the total number of productive suckers, the number of plants with ears and the length of the ears. The grain yield of the first season was zero because of a rainfall deficit but that of the biomass was encouraging $(10,96$ tha- 1$)$ Thus, soil biomechanical design, accompanied by a good organo-mineral fertilizers and good agricultural practices is an effective method for the reclamation and valorization of saline lands. However, this experience deserves to be experienced with other short cycle improved rice varieties.

Keywords: Fatick, Ndoff, salinity, acidity, biomechanics, recovery, rice.

\section{INTRODUCTION}

La salinisation et l'acidification des terres sont des contraintes majeures pour la riziculture qui occupe une place importante dans l'économie du Sénégal (Diaw, 2000). Elles affectent environ $33 \%$ des terres émergées de la région de Fatick selon Chauvin (2013). La production mondiale de riz est dominée par l'Asie avec $91 \%$ suivie de l'Amérique 3,6\% et de l'Afrique 2,9\% (Ahmadi et al., 2014). Le riz est une des principales cultures céréalières qui constitue la base alimentaire de près du tiers de la population mondiale (FAO, 2012). Environ 140 millions d'hectares sont consacrés à la culture du riz dans le monde (FAO, 2012). Le riz est une denrée très consommée au Sénégal. II est cultivé

\section{MATÉRIEL ET MÉTHODES}

Site d'étude : Le village d'intervention est Ndoff. II est situé dans la partie Est de la commune rurale de Loul Sessène, région de Fatick. Le site d'étude se trouve dans le domaine fluviomarin du Sine- Saloum (200 000 hectares). Les coordonnées géographiques du village sont $14^{\circ} 15^{\prime} 3,37^{\prime \prime}$ de latitude nord et $14^{\circ} 24^{\prime} 39,90^{\prime \prime}$ de longitude ouest. La pluviométrie moyenne se situe entre 500 et $600 \mathrm{~mm}$ avec des températures moyennes sur toutes les positions topographiques, et notamment dans les bas-fonds où le potentiel de rendement estimé entre 5 et 6 tha $^{-1}$ est supérieur à celui des plateaux qui est de l'ordre de 2 à 3 tha1 (Sow, 1996). La riziculture pluviale occupe plus de la moitié du potentiel de terres rizicultivables (Ndiaye, 2013). Cette riziculture est très touchée par la salinisation surtout dans le bassin du fleuve Sine-Saloum particulièrement la région de Fatick (Sadio, 1991). La présente étude cherche à étudier les effets du dispositif biomécanique sur les sols salés et la culture du riz à Ndoff dans le bassin fluvio-marin du Sine-Saloum.

oscillant entre de 20 à $35^{\circ} \mathrm{C}$ (ISRA, 2002). La longueur moyenne de la saison des pluies qui débute en juin et se termine en octobre est de cent (100) jours. Les sols ferrugineux et les sols halomorphes ou «tannes» sont les grands types de sol rencontrés dans la zone (Diangar, 2006)

Matériel végétal : Trois (03) variétés de riz et trois (03) espèces végétales de fixation des diguettes ont été 

culture du riz dans le bassin fluvio-marin du Sine-Saloum à Ndoff (Sénégal).

utilisées pour l'essai variétal. II s'agit d'une variété locale et de deux variétés améliorées. Ainsi, dans le site d'expérimentation de Ndoff, la variété locale proposée de manière consensuelle par les producteurs de riz est la variété « Diayi ». Les variétés améliorées choisies par l'équipe de recherche sont «Rock 5 » et « War 77 ». La « Rock 5 » est une variété à cycle long, résistante à la salinité et aux insectes (ISRA, 2012). Elle présente une forte sensibilité à l'égrenage avec un rendement potentiel en grains de 5 tha $^{-1}$. La «War 77 » est également une variété à cycle long, résistante à la salinité et aux maladies comme la pyriculariose. La variété « War 77 » est sensible à l'acidité, son potentiel de grain rendement est de 2 à 3 tha- ${ }^{-1}$ et son poids des 1000 graines est de 27 grammes (ISRA, 2012). En outre, sur la digue principale marquant le pourtour du dispositif expérimental en split plot est planté Vetiveria zizanioides, et autour des diguettes, Gossypium hirsutum et Andropogon gayanus. ont été utilisées comme matériel végétal.

\section{Dispositif et conduite de l'essai}

Prélèvement d'échantillons de sols dans les parcelles : Les caractéristiques physico-chimiques des sols prélevés ont été déterminées au laboratoire. Les échantillons de sols ont été prélevés à l'aide d'une tarière. Toutes les parcelles élémentaires aménagées (09) et les témoins hors du dispositif ont fait l'objet de prélèvements avant semis et après récolte $(0-25 \mathrm{~cm})$ ce qui fait total 24 échantillons. Après ce sondage dans les 25 premiers $\mathrm{cm}$, une analyse du sol dans tout son profil a été effectuée. Les résultats des analyses et ceux de l'étude morphologique ont permis de déterminer les caractéristiques physico-chimiques exactes du sol sur lequel repose l'essai.En effet, un échantillon composite de sol a été recueilli au niveau de chaque parcelle prospectée. II s'agit de Six (06) prélèvements élémentaires suivant les diagonales mélangées soigneusement à l'aide d'une bassine afin d'obtenir cet échantillon composite (Brady, 2014).

L'emplacement des fosses pédologiques, le fonçage et la description des profils pédologiques : L'emplacement a été défini suivant la topovariance dans la tanne enherbée en tenant également en compte la couverture végétale et la géomorphologie. Deux fosses (02) pédologiques ont été creusées au niveau de la zone, l'une sur terrasse basse dans la tanne enherbée et l'autre en terrasse haute dans la tanne arbustive. Chaque fosse a une longueur de 1,5 $\mathrm{m}$, une largeur de $1 \mathrm{~m}$ et une profondeur de $1,5 \mathrm{~m}$. Tous les horizons de chaque profil ont été prélevés à l'aide d'un piochon. La description des caractères morphologiques et biologiques des profils pédologiques a été effectuée pour les paramètres suivants: la couleur, la matière organique, l'humidité, la texture, la structure, la porosité, la consistance du matériau, les traces d'activité biologique, les racines et les inclusions (photo 1). La synthèse de toutes ces informations a permis de donner une proposition de classification du sol étudié. Cette classification sera confirmée ou infirmée après exploitation des données d'analyse des sols.

Conduite culturale : elle a été réalisée avec les producteurs. Après préparation du sol, la fumure organique (fumier) a été apportée $(250 \mathrm{~kg} /$ parcelle). Le labour traditionnel a été fait à l'aide d'un "Gobby ». Avant l'enfouissement et le nivellement du sol, un apport d'engrais minéral a été appliqué à la dose de $150 \mathrm{~kg} / \mathrm{ha}$. Le semis a été effectué manuellement suivant des écartements de $50 \mathrm{~cm}$ entre les lignes et sur la ligne. Aucun traitement phytosanitaire n'a été réalisé. La lutte contre les mauvaises herbes a été manuelle. En ce qui concerne l'entretien des cultures, le démariage a été effectué 15 jours après la levée (JAL) avec 4 plants par poquet. Un premier sarclage a été fait $7 \mathrm{JAL}$ et un 2ème à $15 \mathrm{JAL}$.

Le dispositif expérimental : Le dispositif expérimental est un split plot. Le facteur principal est l'espèce de reboisement (fixation des diguettes) et le facteur secondaire est la variété de riz. Les variables de levée, de survie, de croissance et de production ont été suivies. Le dispositif expérimental est constitué de 3 répétitions (Rep1, Rep2 et Rep3), chaque répétition comprend 3 parcelles et chaque parcelle est constituée de 3 sous-parcelles ou parcelles élémentaires. La parcelle expérimentale est un carré de $54 \mathrm{~m}$ de côté ce qui équivaut à une superficie de $2916 \mathrm{~m}^{2}$. Chaque variété occupe une sous-parcelle de $5 \mathrm{~m} \times 15 \mathrm{~m}$ soit une superficie de $75 \mathrm{~m}^{2}$. Chaque parcelle comprend 26 lignes avec des écartements de $0,5 \mathrm{~m}$ entre les lignes et $0,5 \mathrm{~m}$ sur les lignes. Ce dispositif expérimental est entouré par une grande diguette d'une hauteur de 60 $\mathrm{cm}$. L'expérimentation a pour but de tester la performance des variétés "Rock 5 » et "War 77 » (variétés améliorées) et "Diayi » (variété locale) en milieu salé et identifier ainsi la ou les variété(s) la ou les plus adaptée(s). 


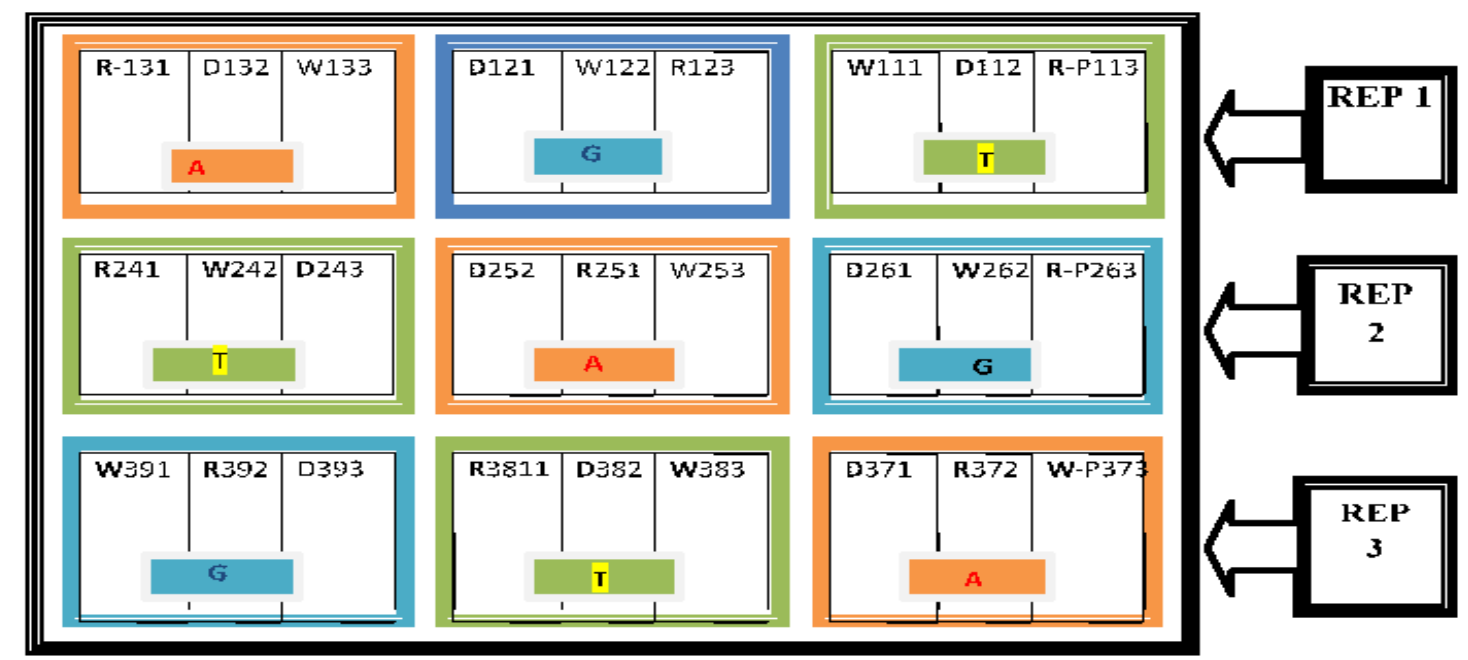

Figure 1 : Schéma du dispositif expérimental pour la culture du riz

La digue principale avec Vetiveria zizanoides

Les parcelles à Andropogon gayanus.

Les parcelles témoins

Les parcelles à Gossypium hirsutum
R : Rock 5

D : Diayi

W : War 77

A : Andropogon gayanus. ; G : Gossypium hirsutum ; $\mathbf{T}$ : Témoin

W111 : War 77 dans la répétition 1 , parcelle 1 et sous parcelle 1

Analyse statistique: Le logiciel Statistix 8.1 a été utilisé pour les analyses de variance afin de vérifier l'existence ou non de différences significatives entre les traitements. Une comparaison de moyennes a été réalisée pour identifier les traitements significativement différents, grâce au test de Turkey et LSD (Least Significant Difference) au seuil de $5 \%$. Les variables agronomiques mesurées sur le riz sont: le taux de levée (TL), l'état des plants (vert, jaune, mort), la hauteur des plants $(H)$, la longueur des épis (LE), le nombre total de talles (NTT), le nombre total de talles productives (NTTP), le nombre total de talles axillaires (NTTA), le nombre de plants avec épis (NPE) et le poids de la matière sèche (PMS). Les paramètres du sol sont consignés dans le tableau ci-dessous. 

culture du riz dans le bassin fluvio-marin du Sine-Saloum à Ndoff (Sénégal).

Tableau 1 : Paramètres du sol analysés

\begin{tabular}{|c|c|}
\hline & \\
\hline Variables & Méthode \\
\hline Conductivité Électrique (CE) & $\begin{array}{l}\text { Extrait aqueux } 1 / 5 \text {, soit } 30 \mathrm{~g} \text { de « terre fine » mélangée à } 150 \mathrm{~mL} \text { d'eau distillée bouillie } \\
\text { puis refroidie à } 25^{\circ} \mathrm{C} \text {. Elle est déterminée sur un conductimètre à mesure directe. }\end{array}$ \\
\hline Granulométrie & $\begin{array}{l}\text { Méthode dite « pipette de Robinson ». La méthode est basée sur la différence de } \\
\text { vitesse de sédimentation entre les particules légères et les plus lourdes. Les fractions } \\
\text { fines (argiles et limons) sont atteintes par des mesures de sédimentation, tandis que } \\
\text { les fractions grossières (sables grossiers, moyens et fins) sont isolées par tamisage sur } \\
\text { des tamis normalisés. }\end{array}$ \\
\hline $\mathrm{pH}$ eau & $\begin{array}{l}\text { Electrométrie sur l'extrait aqueux } 1 / 2,5 \text { soit } 20 \mathrm{~g} \text { de « terre fine » mélangée à } 50 \mathrm{~mL} \\
\text { d'eau distillée, bouillie et refroidie. Le pH est déterminé sur un pH-mètre à lecture } \\
\text { directe }\end{array}$ \\
\hline $\mathrm{pH} \mathrm{KCl}$ & $\begin{array}{l}\text { La mesure du Ph } \mathrm{KCl} \text { se fait sur une solution saline obtenue après addition de } 3,75 \mathrm{~g} \\
\text { de chlorure de potassium } \mathrm{KCl} \text { dans la suspension ayant servi à la détermination du } \\
\text { pHeau. La valeur obtenue est le plus souvent inferieur à celle donnée par la mesure du } \\
\text { pHeau et exprime la capacité des ions } \mathrm{K}^{+} \text {à se substituer aux ions } \mathrm{H}^{+} \text {. II est déterminé } \\
\text { sur un pH-mètre à lecture directe. }\end{array}$ \\
\hline Carbone total & $\begin{array}{l}\text { Walkley-Black modifiée avec un dosage par colorimétrie au spectrophotomètre à la } \\
\text { longueur d'onde } \Lambda=600 \mu \mathrm{m} \text {. }\end{array}$ \\
\hline Azote total & $\begin{array}{l}\text { Méthode Kjeldahl modifiée. L'ammonium est déterminé au spectrophotomètre UV Vis } \\
6705 \text { à la longueur d'onde } \lambda=660 \mathrm{~nm} \text {. }\end{array}$ \\
\hline Phosphore assimilable & Bray I \\
\hline $\begin{array}{l}\text { Capacité d'Échange Cationique } \\
\text { (CEC). }\end{array}$ & $\begin{array}{l}\text { La méthode au chlorure cobaltithexamine. Après lessivage avec une solution de } \\
\text { lanthane, ils sont dosés par absorption atomique pour les cations métalliques }\left(\mathrm{Ca}^{2+} \text { et }\right. \\
\left.\mathrm{Mg}^{2+}\right) \text { et par photométrie à flamme pour les cations sodi-potassiques }\left(\mathrm{Na}^{+} \text {et } \mathrm{K}^{+}\right) \text {. Le } \\
\text { dosage de la CEC s'effectue par colorimétrie automatique de l'ion ammonium au } \\
\text { Dichloroisocyanurate de Sodium. }\end{array}$ \\
\hline & Paramètres calculés \\
\hline Le rapport C/N & Calculé \\
\hline Indice de Stabilité Structurale & $\begin{array}{l}\text { Calculé par la formule suivante selon Pieri, cité par (Dugué.et al., 2002) : St }=(\mathrm{MO} \% \text { * } \\
100) /(\mathrm{A}+\mathrm{L}) \% \text {. Quand la structure devient trop instable, les rendements s'effondrent. } \\
\text { La structure serait dégradée pour } \mathrm{St}<5 \text { et bonne pour } \mathrm{St}>9 \text {. }\end{array}$ \\
\hline
\end{tabular}

\section{RÉSULTATS}

\section{Les données du sol ayant abrité l'essai}

Synthèse des caractéristiques morphologiques et analytiques du sol : La prospection pédologique dans le site a permis d'identifier une seule unité de sol (US) : Sol hydromorphe minéral, salé, sulfaté acide, sur matériau d'ensemble sableux alluvio-colluvionnaire. Ce type de sol est recouvert d'un tapis herbacé essentiellement constitué de Eragrostis squamata, Leptadaenia hastata et Spermacoce verticillata. Le profil du sol présente quelques plages à orientation verticale de couleur jaune-citron et ocre jaune en semiprofondeur et en profondeur. Le sol est caractérisé par un horizon humifère à peu humifère dans les horizons superficiels. II est victime d'un éboulement du matériau en profondeur. Dans la première moitié du profil, on note la présence d'un horizon ocre-rougeâtre et ocre- jaunâtre (forte accumulation d'oxyde de fer) ce qui dénote de la pédogenèse complexe de ce sol. Le matériau sableux dunaire a beaucoup influencé la mise en place de ce sol. II présente une texture d'ensemble sableuse $(92,2 \%)$ à dominance de sables moyens $(84,15 \%)$. La structure de ce sol est très dégradée (st $=1,005)$. Ce sol, situé en terrasse basse, est très salé dans l'ensemble du dispositif au niveau des 25 premiers $\mathrm{cm}(\mathrm{CE}=4,013 \mathrm{mS} / \mathrm{cm})$ et très acide $(\mathrm{pH}$ eau $=$ 4,3). Cependant, le sol devient moins salé (CE $=1,6$ $\mathrm{mS} / \mathrm{cm})$ et moins acide $\left(\mathrm{pH}_{\text {eau }}=4,5\right)$ en profondeur. II est très pauvre en matière organique $(0,08 \%)$. Cette dernière se minéralise très lentement car le rapport $\mathrm{C} / \mathrm{N}$ est très élevé $(26,7)$. Un taux moyen de phosphore assimilable est noté (45 ppm). C'est un sol moyennement pourvu en azote $(0,2 \%)$. En revanche, 
il est bien pourvu en potassium $(3,66 \mathrm{meq} / 100 \mathrm{~g}$ de sol). La capacité d'échange cationique (CEC) est jugée très faible $(3,5 \mathrm{meq} / 100 \mathrm{~g}$ de sol) tandis que le taux de sodium échangeable (ESP ou $\mathrm{Na}^{\star} 100 / \mathrm{T}$ ) du sol est en moyenne de $9,15 \%$ ce qui signifie le sol sol est menacé par le processus de la salinisation. En outre, un taux de saturation en bases très élevé a été noté $(244,10 \%)$ ce qui veut dire le sol est trop saturé en bases. L'analyse des équilibres entre les principaux éléments nutritifs du sol a donné un rapport de 1/3,55 pour $\mathrm{K} / \mathrm{Mg}$. Ce rapport entre les teneurs en $\mathrm{K} / \mathrm{Mg}$ indique un excès en magnésium dans le sol. Cependant, la valeur absolue $\mathrm{k} / \mathrm{Ca}$ indique un excès $\mathrm{du}$ potassium par rapport au calcium. Ainsi, l'excès du potassium dans ce milieu par rapport aux teneurs en Magnésium et calcium pourrait nuire la nutrition minérale de la plante du riz $\mathrm{Mg}$ et $\mathrm{Ca}$. La valeur élevée du taux de saturation en bases serait due aux fortes teneurs du sol en potassium. C'est un sol très saturé en bases notamment en $\mathrm{K}^{+}$. 
Seye et al., J. Appl. Biosci. 2018 Effet d'un dispositif biomécanique sur la récupération de sols salés et la culture du riz dans le bassin fluvio-marin du Sine-Saloum à Ndoff (Sénégal).

Tableau 2 : Résultats des analyses du sol $(0-25 \mathrm{~cm})$

$$
\text { Avant semis }
$$

\begin{tabular}{|c|c|c|c|c|c|c|c|c|c|c|c|c|c|c|c|c|c|c|c|c|}
\hline \multicolumn{21}{|c|}{ Avant semis } \\
\hline & & \multicolumn{2}{|c|}{$\mathrm{pH}$} & $\mathrm{CE}$ & \multicolumn{4}{|c|}{ Matière organique } & \multicolumn{7}{|c|}{ Bases échangeables } & \multicolumn{5}{|c|}{ Granulométrie (\%) } \\
\hline Répétitions & Espèces & $\begin{array}{l}\mathrm{pH} \\
\text { eau }\end{array}$ & $\mathrm{pH} \mathrm{Kcl}$ & $\begin{array}{l}\text { CE } \\
(\mu S / \mathrm{cm})\end{array}$ & $\begin{array}{l}\mathrm{P}_{2} \mathrm{O}_{5} \\
(\mathrm{ppm})\end{array}$ & C \% & Nt $\%$ & $\mathrm{C} / \mathrm{N}$ & $\begin{array}{l}\mathrm{Ca}^{2+} \\
\mathrm{meq} / 100 \mathrm{~g}\end{array}$ & $\begin{array}{l}\mathrm{Mg}^{2+} \\
\text { meq/ } \\
100 \mathrm{~g}\end{array}$ & $\begin{array}{l}\mathrm{Na}^{+} \\
\text {meq/ } \\
100 \mathrm{~g}\end{array}$ & $\begin{array}{l}\mathrm{K}^{+} \\
\text {meq/ } \\
100 \mathrm{~g}\end{array}$ & $\begin{array}{l}\mathrm{S} \\
\text { meq/ } \\
100 \mathrm{~g}\end{array}$ & $\begin{array}{l}\mathrm{T} \\
\text { meq/ } \\
100 \mathrm{~g}\end{array}$ & V \% & Argile & Limon & $\begin{array}{l}\text { sables } \\
\text { fins }\end{array}$ & $\begin{array}{l}\text { Sable } \\
\text { moyen }\end{array}$ & $\begin{array}{l}\text { Sable } \\
\text { grossier }\end{array}$ \\
\hline 1 & $\mathrm{~T}$ & 4,30 & 4,10 & 2252 & 18,93 & 3,49 & 0,12 & 28,59 & 6,59 & 1,14 & 0,17 & 2,73 & 10,63 & 2,74 & 387,39 & 3,07 & 2,83 & 1,06 & 87,35 & 5,45 \\
\hline 1 & $\mathrm{GH}$ & 4,40 & 4,10 & 2545 & 12,45 & 4,37 & 0,16 & 27,29 & 1,86 & 1,83 & 0,53 & 2,72 & 6,94 & 3,11 & 222,88 & 3,30 & 2,85 & 1,40 & 85,85 & 6,30 \\
\hline 1 & AG & 4,40 & 4,10 & 1980 & 10,96 & 4,64 & 0,19 & 24,17 & 1,14 & 0,54 & 0,45 & 2,67 & 4,80 & 3,06 & 156,88 & 3,22 & 3,33 & 1,45 & 86,95 & 5,20 \\
\hline 1 & $\mathrm{TH}$ & 4,00 & 4,00 & 4590 & 19,00 & 3,38 & 0,12 & 28,15 & 6,80 & 1,60 & 0,69 & 6,79 & 15,87 & 5,49 & 289,30 & 6,80 & 3,97 & 1,85 & 82,30 & 5,30 \\
\hline 2 & $\mathrm{~T}$ & 4,30 & 4,00 & 6239 & 8,97 & 5,30 & 0,22 & 27,60 & 3,23 & 1,84 & 0,22 & 1,31 & 6,60 & 3,22 & 205,13 & 4,10 & 3,92 & 1,30 & 84,90 & 6,20 \\
\hline 2 & $A G$ & 4,20 & 4,10 & 3244 & 10,46 & 4,15 & 0,17 & 24,39 & 1,97 & 1,22 & 0,12 & 1,29 & 4,61 & 3,27 & 140,86 & 4,37 & 3,00 & 1,90 & 85,90 & 5,25 \\
\hline 2 & $\mathrm{GH}$ & 4,30 & 4,10 & 3686 & 14,44 & 3,76 & 0,16 & 23,96 & 2,28 & 1,64 & 0,33 & 5,55 & 9,80 & 3,22 & 304,48 & 4,42 & 3,13 & 1,35 & 83,25 & 8,00 \\
\hline 2 & $\mathrm{TH}$ & 4,30 & 4,10 & 3078 & 14,44 & 4,75 & 0,17 & 28,11 & 1,18 & 1,51 & 0,25 & 3,43 & 6,36 & 3,96 & 160,74 & 4,85 & 2,95 & 2,50 & 81,85 & 7,85 \\
\hline 3 & GH & 4,30 & 3,80 & 4996 & 16,44 & 4,59 & 0,16 & 28,84 & 2,75 & 1,16 & 0,28 & 3,43 & 7,61 & 2,69 & 282,94 & 5,95 & 3,85 & 2,15 & 79,80 & 8,80 \\
\hline 3 & $\mathrm{~T}$ & 4,40 & 4,10 & 3573 & 20,42 & 4,75 & 0,17 & 27,95 & 2,74 & 1,71 & 0,24 & 6,79 & 11,49 & 4,80 & 239,27 & 5,42 & 2,90 & 1,15 & 84,95 & 5,95 \\
\hline 3 & $A G$ & 4,50 & 4,10 & 6086 & 10,46 & 6,12 & 0,22 & 28,48 & 3,73 & 2,57 & 0,28 & 3,81 & 10,39 & 2,80 & 371,74 & 5,05 & 3,50 & 1,65 & 83,80 & 6,30 \\
\hline 3 & $\mathrm{TH}$ & 4,20 & 4,10 & 5891 & 9,46 & 5,25 & 0,19 & 28,05 & 0,88 & 1,73 & 0,27 & 3,49 & 6,37 & 3,80 & 167,69 & 5,22 & 3,53 & 1,30 & 82,60 & 7,70 \\
\hline \multicolumn{21}{|c|}{ Après récolte } \\
\hline 1 & $T$ & 5,10 & 5,00 & 2171 & 35,92 & 3,99 & 0,51 & 7,91 & 6,59 & 1,14 & 0,17 & 2,73 & 10,63 & 3,11 & 341,45 & 3,07 & 2,83 & 1,06 & 87,35 & 5,45 \\
\hline 1 & $\mathrm{GH}$ & 5,20 & 4,90 & 1880 & 42,50 & 3,16 & 0,20 & 15,64 & 1,14 & 0,54 & 0,05 & 2,72 & 4,45 & 3,06 & 145,51 & 3,30 & 2,85 & 1,40 & 85,85 & 6,30 \\
\hline 1 & $A G$ & 5,30 & 5,00 & 4078 & 77,22 & 6,08 & 0,28 & 21,89 & 1,86 & 1,83 & 0,42 & 2,67 & 6,77 & 2,74 & 246,88 & 3,22 & 3,33 & 1,45 & 86,95 & 5,20 \\
\hline 1 & $\mathrm{TH}$ & 5,10 & 4,90 & 2852 & 24,54 & 4,83 & 0,18 & 27,32 & 6,80 & 1,60 & 0,69 & 6,79 & 15,87 & 5,49 & 1404,2 & 6,80 & 3,97 & 1,85 & 82,30 & 5,30 \\
\hline 2 & $\mathrm{~T}$ & 5,30 & 4,90 & 3558 & 50,28 & 3,99 & 0,28 & 23,48 & 1,14 & 1,84 & 0,22 & 1,31 & 6,60 & 3,22 & 205,13 & 4,10 & 3,92 & 1,30 & 84,90 & 6,20 \\
\hline 2 & $A G$ & 5,20 & 4,80 & 2125 & 38,91 & 3,16 & 0,21 & 19,65 & 0,54 & 1,22 & 0,12 & 1,29 & 4,61 & 3,27 & 140,86 & 4,37 & 3,00 & 1,90 & 85,90 & 5,25 \\
\hline 2 & $\mathrm{GH}$ & 5,30 & 5,00 & 1941 & 43,10 & 6,08 & 0,20 & 28,04 & 1,83 & 1,64 & 0,33 & 5,55 & 9,80 & 3,22 & 304,48 & 4,42 & 3,13 & 1,35 & 83,25 & 8,00 \\
\hline 2 & $\mathrm{TH}$ & 5,00 & 4,70 & 1768 & 28,14 & 2,98 & 0,25 & 11,81 & 1,18 & 1,51 & 0,25 & 3,43 & 6,36 & 3,96 & 160,74 & 4,85 & 2,95 & 2,50 & 81,85 & 7,85 \\
\hline 3 & $\mathrm{GH}$ & 5,30 & 5,10 & 2757 & 37,71 & 4,41 & 0,38 & 11,65 & 1,71 & 1,71 & 0,28 & 3,16 & 7,89 & 2,69 & 293,42 & 5,95 & 3,85 & 2,15 & 79,80 & 8,80 \\
\hline 3 & $\mathrm{~T}$ & 5,30 & 4,90 & 2135 & 43,10 & 4,35 & 0,15 & 28,73 & 2,57 & 2,57 & 0,42 & 7,17 & 12,73 & 2,80 & 1698,2 & 5,42 & 2,90 & 1,15 & 84,95 & 5,95 \\
\hline 3 & $A G$ & 4,80 & 5,00 & 4610 & 68,24 & 6,20 & 0,23 & 27,29 & 1,16 & 1,16 & 0,28 & 3,81 & 6,41 & 4,80 & 681,48 & 5,05 & 3,50 & 1,65 & 83,80 & 6,30 \\
\hline 3 & $\mathrm{TH}$ & 5,00 & 4,70 & 2662 & 55,07 & 5,01 & 0,20 & 24,79 & 0,88 & 1,73 & 0,28 & 3,49 & 6,38 & 3,80 & 167,90 & 5,22 & 3,53 & 1,30 & 82,60 & 7,70 \\
\hline
\end{tabular}

Tableau 3: Résultats des analyses du profil du sol

\begin{tabular}{|c|c|c|c|c|c|c|c|c|c|c|c|c|c|}
\hline Noms & horizons $(\mathrm{cm})$ & $\mathrm{CE}(\mu \mathrm{S} / \mathrm{cm})$ & $\mathrm{pH}_{\text {eau }}$ & $\mathrm{pH} \mathrm{KCl}$ & $\mathrm{P}$ (ppm) & $\begin{array}{l}\text { Carbone total } \\
(\%)\end{array}$ & \begin{tabular}{|l|} 
Azote total \\
$(\%)$
\end{tabular} & $\mathrm{C} / \mathrm{N}$ & $\begin{array}{l}\text { Argiles } \\
(\%)\end{array}$ & Limons $\quad(\%)$ & $\begin{array}{ll}\begin{array}{l}\text { sables } \\
(\%)\end{array} \\
\end{array}$ & \begin{tabular}{|l|} 
Sables \\
moyens (\%)
\end{tabular} & \begin{tabular}{|l|} 
Sables \\
grossiers (\%)
\end{tabular} \\
\hline $\begin{array}{l}\text { SSP2 } \\
\text { SSP2 } \\
\text { SSP2 } \\
\text { SSP2 }\end{array}$ & $\begin{array}{l}0-15 \\
15-55 \\
55-130 \\
130-150\end{array}$ & $\begin{array}{l}1515 \\
740.1 \\
1694 \\
1776\end{array}$ & $\begin{array}{l}4,7 \\
4,6 \\
4,4 \\
4,4\end{array}$ & $\begin{array}{l}4,6 \\
4,3 \\
4,2 \\
4,2\end{array}$ & $\begin{array}{l}22,748 \\
10,177 \\
1,197 \\
0,599\end{array}$ & \begin{tabular}{|l|}
3,635 \\
0,775 \\
0,656 \\
0,656
\end{tabular} & \begin{tabular}{|l|}
0,303 \\
0,09 \\
0,252 \\
0,085
\end{tabular} & $\begin{array}{l}12 \\
8,59 \\
2,6 \\
7,7\end{array}$ & $\begin{array}{l}4,15 \\
4,62 \\
8,12 \\
7,65\end{array}$ & $\begin{array}{l}4,02 \\
1,45 \\
2,43 \\
1,6\end{array}$ & $\begin{array}{l}1,4 \\
0,45 \\
1,55 \\
1,35\end{array}$ & $\begin{array}{l}83,15 \\
87,65 \\
74,4 \\
76,5\end{array}$ & \begin{tabular}{|l|}
8 \\
5,6 \\
12,8 \\
12,3
\end{tabular} \\
\hline
\end{tabular}


Dynamique des paramètres physico-chimiques du sol avant semis et après récolte: L'analyse de variance a été appliquée à toutes les variables du sol. Seule la capacité d'échange cationique a présenté des effets significatifs en fonction des espèces de fixation des diguettes. La conductivité électrique et le $\mathrm{pH}$ eau ont par contre révélé des effets significatifs en fonction de la période (avant semis et après récolte) (tableau 4).

Tableau 4 : Analyse de variance sur les paramètres du sol

\begin{tabular}{l|c|c|c|c|c|c}
\hline \multicolumn{1}{c}{ Source de variation } & Variables & DDL & SC & CM & F & P \\
\hline Espèces de fixation & $\begin{array}{c}\text { capacité d'échange } \\
\text { cationique (T) }\end{array}$ & 3 & 7,894 & 2,631 & 4,390 & 0,022 \\
\hline Période & $\begin{array}{c}\text { conductivité électrique } \\
\text { (CE) }\end{array}$ & 1 & 1,017 & 1,010 & 6,200 & 0,003 \\
\hline Période & pHeau & 1 & 4,420 & 4,420 & 216,510 & $<0,001$ \\
\hline
\end{tabular}

La Conductivité électrique : Les résultats du test de comparaison de moyennes par LSD (Least Significant Difference) ont montré deux groupes homogènes $A$ et B. Le groupe A (avant semis) a présenté une moyenne significativement différente de celle du groupe $B$ (après récolte). La moyenne de $\mathrm{CE}$ a significativement diminué dans la période après récolte passant de 4013,3 à $2711,43 \mu \mathrm{S} . \mathrm{cm}-1$.

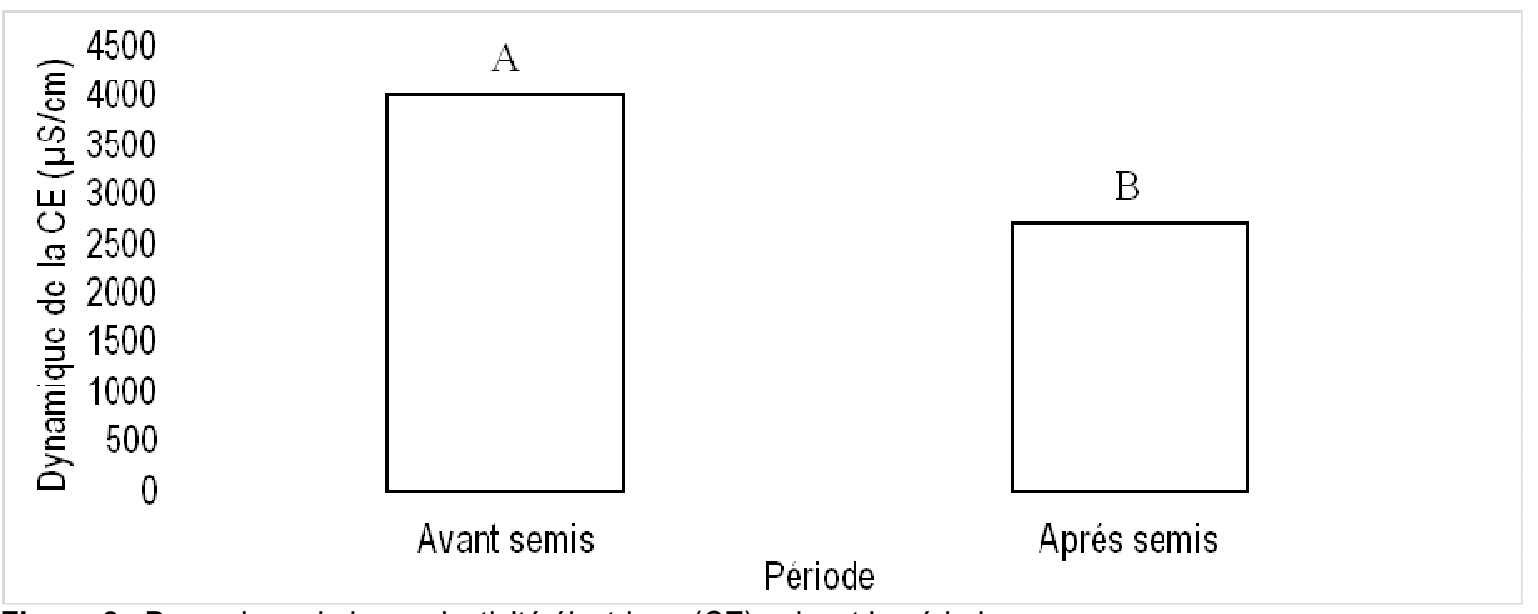

Figure 2 : Dynamique de la conductivité électrique (CE) suivant la période

Le $\mathrm{pH}$ eau: La figure 3 montre deux périodes homogènes $A$ et $B$. Le groupe homogène $A$ (avant semis) a présenté une moyenne significativement différente de celle du groupe $B$ (après récolte). La moyenne du $\mathrm{pH}$ eau $(4,3)$ très acide dans la période avant semis a considérablement augmenté dans la période après récolte avec un $\mathrm{pH}_{\text {eau }}=5,2$ d'après le test de comparaison de moyenne par LSD (Least Significant Difference). 


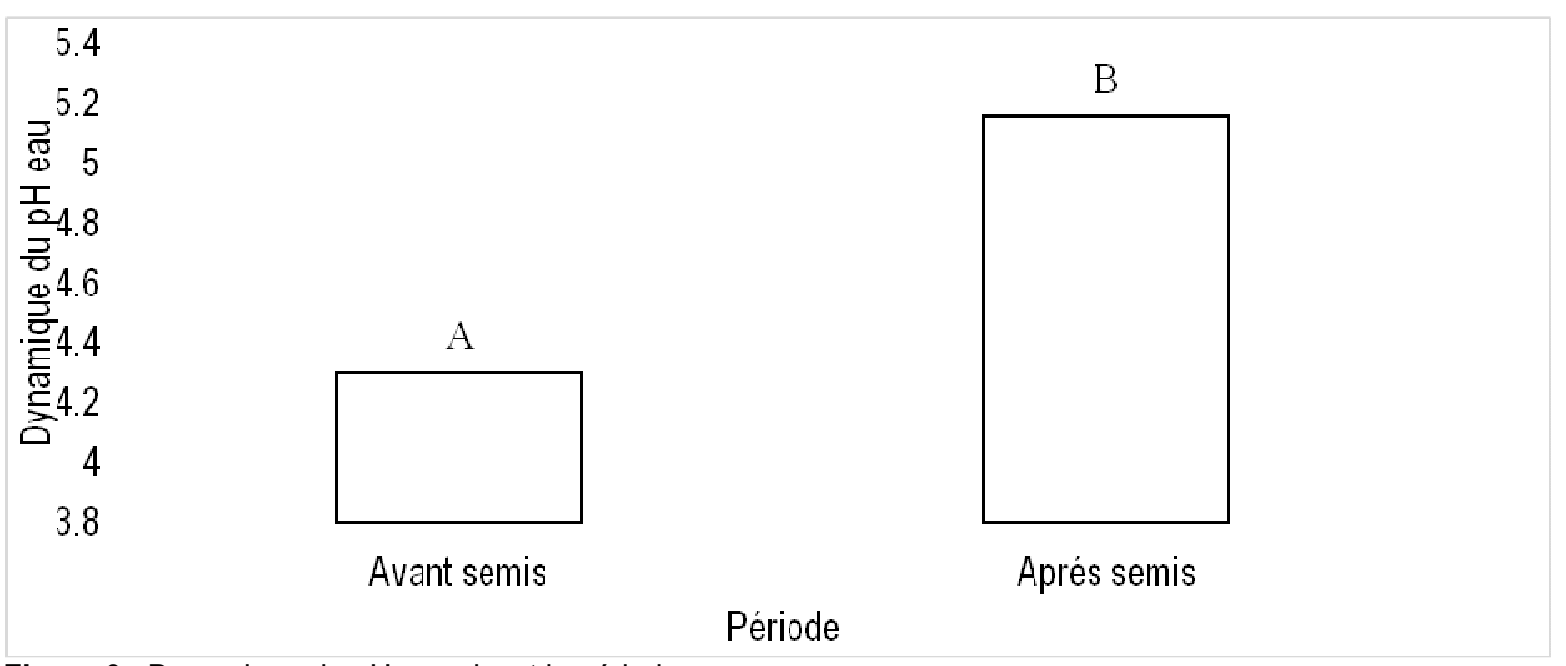

Figure 3 : Dynamique du $\mathrm{pH}_{\text {eau }}$ suivant la période

La capacité d'échange cationique $(T)$ : Les résultats du test Tukey de la figure 4 ont révélé trois groupes homogènes $(A$ et $B)$ et un groupe intermédiaire $A B$. Le groupe A est représenté par le témoin hors du dispositif (TH). Gossypium hirsutum (GH) et le témoin $(\mathrm{T})$ ont constitué le groupe $B$. Le groupe $(A B)$ est représenté par Andropogon gayanus.. Le témoin hors du dispositif $(\mathrm{TH})$ a enregistré en moyenne une capacité d'échange cationique $(T)$ plus élevée que celle du groupe $B$ (Gossypium hirsutum et le témoin) et du groupe $A B$ (Andropogon gayanus.).

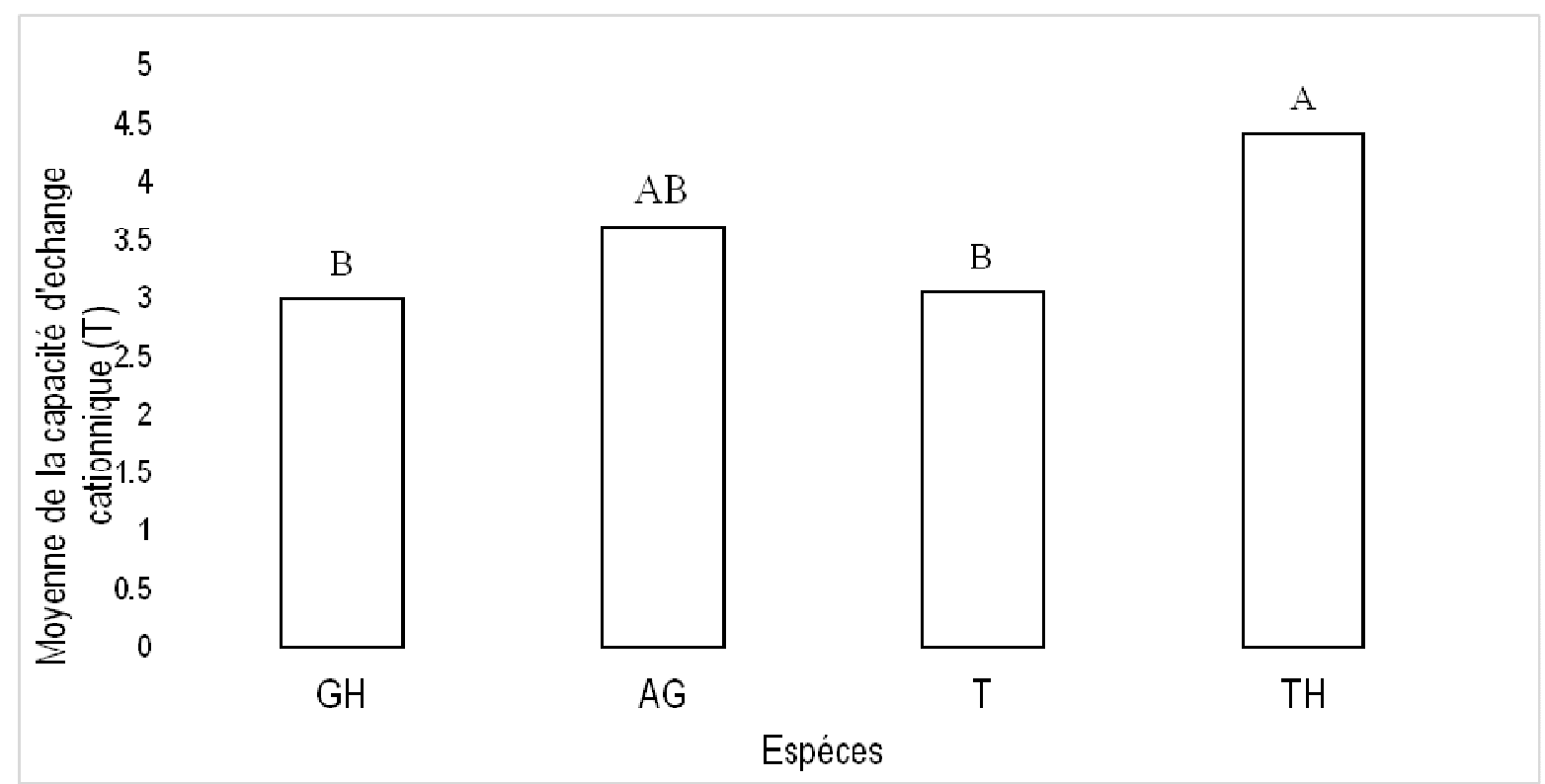

Figure 4 : Variation moyenne de la capacité d'échange cationique $(T)$

$\mathrm{GH}=$ Gossipium hirsutum ; $\mathrm{AG}=$ Andropogon gayanus. ; T = Témoin aménagé ; TH = Témoin hors du dispositif

Les résultats agronomiques: L'analyse de variance sur la longueur des épis, le nombre de plants avec épis, le nombre de talles productives, l'état jaune ou vert des plants a révélé des différences significatives (tableau 2). Les tests de comparaison de moyenne par Tukey ont été effectués. Le rendement (Kg.ha-1) est égal à zéro mais le poids de la matière sèche (biomasse) a été évalué bien vrai qu'il n'a pas présenté de différences significatives entre les espèces de fixation des diguettes, les variétés et leurs interactions (espèces et variétés). 
Seye et al., J. Appl. Biosci. 2018 Effet d'un dispositif biomécanique sur la récupération de sols salés et la culture du riz dans le bassin fluvio-marin du Sine-Saloum à Ndoff (Sénégal).

Tableau 5 : Analyse de variance sur les variables du riz

\begin{tabular}{l|l|c|c|c|c|c}
\hline Source de variation & \multicolumn{1}{c|}{ Variables } & DDL & SC & CM & F & P \\
\hline Espèces de fixation & Longueur des épis & 2 & 46,222 & 23,111 & 26,000 & 0,0051 \\
Variété & Longueur des épis & 2 & 832,570 & 416,290 & 22,170 & $<0,001$ \\
Aménagement*Variétés & Longueur des épis & 4 & 272,180 & 68,044 & 3,620 & 0,037 \\
Espèces de fixation & Nombre de plants avec épis & 2 & 1684,670 & 842,330 & 28,290 & 0,004 \\
Variété & Nombre de plants avec épis & 2 & 5513,560 & 2756,800 & 61,110 & $<0,001$ \\
Aménagement*Variétés & Nombre de plants avec épis & 4 & 2039,780 & 509,940 & 11,300 & $<0,001$ \\
Variétés & Nombre de thalles productifs & 2 & 44,963 & 22,482 & 10,030 & 0,003 \\
Espèces de fixation & Etat vert des plants & 2 & 46,222 & 23,111 & 26,000 & 0,005 \\
Espèces de fixation & Etat jaune des plants & 2 & 60,222 & 30,111 & 11,060 & 0,023 \\
Espèces de fixation & Poids de la matière sèche & 2 & 735139 & 367569 & 5,000 & 0,082 \\
Variétés & Poids de la matière sèche & 2 & 290556 & 145278 & 1,530 & 0,225 \\
Aménagement*variétés & Poids de la matière sèche & 4 & 180972 & 45243 & 0,480 & 0,752 \\
\hline
\end{tabular}

\section{DISCUSSION}

II est important de rappeler que la vallée de Ndoff où est conduite l'expérimentation se situe dans le bassin fluviomarin du Sine-Saloum. Cette vallée est reliée à une série de marigots qui sont des affluents du fleuve Sine-Saloum. Ce bassin a connu peu d'études sur les sols. Cette présente étude a été menée à l'échelle locale pour étudier les effets du dispositif biomécanique sur les sols salés et sur la culture de trois variétés de riz (une locale et deux améliorées).

Effets du dispositif biomécanique sur les sols salés: La dynamique des caractéristiques physicochimiques du sol: Le but visé est d'étudier l'effet du dispositif biomécanique à travers la dynamique des caractéristiques physico-chimiques du sol avant semis et après récolte. En effet, les résultats ont montré d'abord une différence significative de la Conductivité électrique (CE) du sol suivant la période. La conductivité électrique (CE) du sol a diminué suivant les deux périodes en passant de 4013,3 (avant semis) à $2711,4 \mu \mathrm{S} / \mathrm{cm}$ (après récolte). Cette baisse de la $\mathrm{CE}$ suivant la période serait due au dispositif biomécanique aménagé dans le site. En effet, le dispositif biomécanique a retenu d'abord les eaux pluviales et a ensuite dilué les sels de la solution du sol ce qui expliquerait cette baisse significative. Ainsi, la CE hors du dispositif est plus élevée que la CE à l'intérieur du dispositif biomécanique. Ceci est dû à un bon drainage permettant l'évacuation des sels dissouts hors du dispositif biomécanique. Ceci est en contradiction avec l'étude de Fall (2002) où la CE à l'intérieur du dispositif est plus élevée qu'à l'extérieur. En outre, après la récolte le $\mathrm{pH}$ eau a augmenté de manière significative suivant la période avant semis $(\mathrm{pH}=4,3)$ et après récolte $(\mathrm{pH}=5,3)$. Cette augmentation du $\mathrm{pH}$ eau serait due aussi d'une part à l'effet important de dilution induit par le dispositif grâce à une accumulation importante des eaux de pluies. En effet, comme le dispositif a été couvert d'eau, les conditions sont devenues peu favorables pour une oxydation de la pyrite (sulfure de fer) dont l'exposition engendre l'acidification du milieu. Le dispositif biomécanique aurait donc empêché alors la libération de l'acide sulfurique responsable de l'acidification du sol dans ce milieu. Parallèlement, les résultats montrent que la capacité d'échange cationique a des effets significatifs en fonction des espèces de fixation des diguettes autrement dit elle est plus élevée hors du dispositif qu'à l'intérieur du dispositif. En effet, ceci s'expliquerait par une minéralisation importante de la matière organique suite à la mise en culture du riz. Notons que la capacité d'échange cationique dépend de la nature de l'argile et de la matière organique (l'humus). II est important d'indiquer que toute zone mise en culture voit sa fertilité diminuer (Baize, 1988). En plus, il a été constaté à l'intérieur du dispositif que la capacité d'échange cationique (CEC) des parcelles aménagées avec Gossypium hirsutum (GH) est beaucoup plus élevée que celles aménagés avec Andropogon gayanus. (AG). Ainsi, Gossypium hirsutum produirait beaucoup plus de matière organique (humus) qu'Andropogon gayanus..

Les contraintes majeures du sol à la production rizicole: La salinisation des sols: Une forte concentration des sels en surface $(0-25 \mathrm{~cm})$ a été mise en évidence. Cette salinité a été appréciée à l'aide des mesures de la CE de l'extrait aqueux 1/5. Globalement, l'existence d'un gradient de salinité croissant a été noté 

culture du riz dans le bassin fluvio-marin du Sine-Saloum à Ndoff (Sénégal).

sur le long du profil du sol mais avec une concentration faible en semi-profondeur ; en même temps, une accumulation importante en profondeur $(150 \mathrm{~cm})$ a été constatée. Cette distribution des sels pourrait s'expliquer par les fluctuations de la nappe phréatique peu profonde. Sous ce rapport, les fortes concentrations de sels en surface sont dues au phénomène de remontée capillaire. La salinisation par remontée capillaire a été démontrée notamment par Marius (1985) et Sadio (1991) qui soutiennent que la salinisation des domaines fluviomarins du Sénégal est due à l'influence marine et à la remontée capillaire. Les faibles concentrations à mi- profondeur seraient dues à l'existence d'un bon drainage hypodermique qui joue un rôle essentiel de lessivage latéral. II existerait donc une nappe perchée saisonnière qui s écoulerait latéralement en emportant les sels. Toutefois, une lixiviation des éléments des horizons de surface vers la profondeur à cause de la texture légère expliquerait le caractère très salé en profondeur.

L'acidification des sols : Les résultats analytiques et morphologiques du sol montrent que les différentes parcelles du dispositif en surface et en profondeur sont très acides. Les valeurs de l'acidité actuelle données par les mesures du $\mathrm{pH}$ eau confirment donc les hypothèses sur l'acidification des sols dans le dispositif. Ainsi, cette acidité serait due à la nature géologique du matériau. Ce dernier est riche en pyrite (FeS), dont l'oxydation donne de la jarosite (sulfate basique de fer). L'hydrolyse de la jarosite entraîne l'acidification (Sadio, 1991). En effet, en conditions anaérobies (saison des pluies), il se produit une réduction des sels sulfatés en pyrite (sulfure de fer). En saison sèche, le ressuyage du sol permet son aération et l'arrivée de l'oxygène provoque l'oxydation de la pyrite en sulfates (jarosite) avec production d'acide sulfurique. Le sol devient ainsi très acide. Ainsi, les taches ocre jaunâtres observées dans le sol témoignent de la présence de jarosite. Ceci a été démontré également par Viellefon (1971,1973 et 1974) cité par Sène (2014) ; Marius (1985) et Sadio (1991). Ainsi, cette acidification est une contrainte majeure pour la croissance et le développement des plants car le riz se comporte mieux si le pH est compris entre 5 et 6,5 (Brady ,2014). En outre, la forte acidité constitue un facteur limitant pour la minéralisation de la matière organique (Sène et al., 2014) car elle inhibe l'activité des bactéries responsables de cette minéralisation. En somme, la solution à l'acidité pourrait être un amendement calcique (chaulage) et la sélection de variétés de riz adaptées et la combinaison engrais minéraux et organique (Larcharme, 2001).
Le faible taux de matière organique : Sur la terrasse basse où est installé le dispositif, les analyses ont révélé un taux de matière organique très faible en surface ainsi qu'en profondeur alors que les rapports $\mathrm{C} / \mathrm{N}$ dans toutes les parcelles sont supérieurs à 20 . Cela signifie que la matière organique se minéralise très lentement; ce qui aurait dû contribuer à une forte accumulation de la matière organique d'où le caractère contradictoire entre le taux de matière organique (MO) et les valeurs du rapport $(\mathrm{C} / \mathrm{N})$. En effet, cela peut s'expliquer d'abord par le caractère hydromorphe du milieu car l'hydromorphie rend faible l'activité biologique notamment du fait des conditions d'anaérobiose et de forte teneur en acide qui y règnent (Sène et al., 2014). Ensuite, la texture sableuse est en phase avec le faible taux de matière organique du fait qu'elle facilite le lessivage de cette dernière. Ceci serait dû au caractère chimiquement peu actif du sable. Enfin, le caractère salé du site pourrait expliquer ce phénomène sachant que les sels solubles entraînent la dispersion des colloïdes (Brady, 2014).

Effet du dispositif biomécanique sur la performance des variétés de riz testées : L'objectif est de tester la performance des trois variétés de riz sous un aménagement biomécanique en milieu salé. Cependant, l'expérimentation a souffert d'une pause pluviométrique car la pluviométrie de Ndoff en 20142015 n'a été que de $387 \mathrm{~mm}$ associée à un $\mathrm{pH}$ très acide $(4,3)$ et une CE très salée $\left(4,013 \mathrm{mS} . \mathrm{cm}^{-1}\right)$. L'arrêt précoce des pluies a été le principal facteur limitant pour les plantes surtout pendant la période de floraison. C'est pourquoi, le rendement à l'issue de cette expérimentation est nul mais la biomasse a été évaluée. Malgré l'arrêt précoce des pluies toutes les variétés de riz ont montré un taux de levée, un nombre total de talles, une croissance en hauteur des plants et une biomasse satisfaisante. D'après l'analyse de variance, toutes ces variables n'ont pas révélé de différence significative. Cependant, la longueur des épis, le nombre de talles productives et le nombre de plants avec épis et l'état des plants (jaune ou vert) ont montré des différences significatives soit en fonction des variétés, soit en fonction de l'espèce de fixation des diguettes et de leur interaction grâce au test de Tukey. Ces résultats amènent à dire que la variété locale « Diayi » s'est mieux comportée pour ne pas dire est plus performante dans les systèmes de production rizicole de Ndoff avec le dispositif aménagé que les variétés «Rock 5 » et «War 77 ». Ceci pourrait s'expliquer par le que fait "Rock 5 » et "War 77 » n'ont pas été utilisées dans leur milieu de prédilection. 
En effet, le type de sol rencontré n'est pas convenable (texture sableuse), la durée du cycle végétatif de ces variétés n'est pas appropriée car elle est longue (ADRAO, 2012). Dans l'expérimentation, c'est le choix des traitements (variétés) qui a fait défaut car au-delà du fait que ces variétés sont très adaptées en milieu

\section{CONCLUSION ET PERSPECTIVES}

Une seule unité de sol hydromorphe minéral, salé, sulfaté acide sur un matériau d'ensemble sableux alluvio-colluvionnaire a été dentifiée à l'intérieur $d u$ dispositif biomécanique. L'acidité et la salinité constituent les principales contraintes à la production rizicole dans la vallée Ndoff. A cela s'ajoutent des contraintes secondaires telles que la texture légère, le faible taux de matière organique et l'excès relatif de magnésium par rapport au potassium. Ainsi, l'étude de la dynamique des paramètres du sol a révélé que le dispositif biomécanique aménagé dans le site joue un rôle déterminant sur la désalinisation et la désacidification du sol car la conductivité électrique (CE) a baissé significativement tout comme le $\mathrm{pH}$ eau qui a significativement augmenté suivant la période avant semis et après récolte (test de comparaison de moyenne par LSD. La dynamique positive de la conductivité électrique (CE) et du $\mathrm{pH}$ montre que le dispositif biomécanique établi a fortement amélioré ces

\section{REFERENCES BIBLIOGRAPHIQUES}

Ahmadi N., Chaterau J. et Ouendeba B., 2014. Les céréales, in « memento », $50 \mathrm{p}$.

ADRAO, 2012. Fiche technique de la riziculture, $16 \mathrm{p}$.

Baize D., 1988. Guide des analyses courantes en pédologie. Paris, $172 \mathrm{p}$.

Brady N.C. \& Weil R. R., 2014.The nature and properties of soils. Dorling Kindersley (India) Pvt. Ltd., fourteenth edition, New Delhi, 1046 p.

Chauvin L., 2013. La salinisation des terres dans la région de Fatick: étendue et conséquences sur les services écosystémiques du système de production agropastoral, mémoire ISE.

Demba F., Savoirs locaux et Conservation de l'agro biodiversité : leçons d'une expérience dans la riziculture traditionnelle dans les villages de Ndoff et de Faoye, mémoire ISE, 2012, 75p.

Dugue P. et Gigou P., 2014. La gestion de la fertilité de la terre in « Memento ».601-614p

Diaw N T., 2000. Evaluation au champs et au condition de salinité aux performance agro morphologiques et physiologiques de lignées salé et acide, elles exigent un minimum pluviométrique et un sol riche en argile et en matière organique qu'on ne trouve pas dans le site d'étude. Cela montre l'importance du choix minutieux de la carte variétale dans le cadre de la récupération des sols salés à des fins agricoles (Montoroi, 1993).

deux caractéristiques physico-chimiques du sol. En outre, les 3 variétés de riz testées n'ont pas décelé de différence significative quant aux taux de levée, à la production de talles, à la hauteur des plants et à la biomasse. Cependant, 95 jours après semis, la variété locale «Diayi», proposée par les producteurs, a obtenu les meilleures moyennes sur la longueur des épis, le nombre de plants épiés et le nombre total de talles productives quand elle est associée au traitement avec Gossypium hirsutum. Ainsi, l'arrêt précoce des pluies a empêché toutes les variétés de boucler leur cycle végétatif. Donc, cette expérience biomécanique de récupération et de valorisation des terres salées associée à l'introduction de variétés adaptées mériterait d'être menée dans d'autres zones agricoles salées du pays ou d'autres pays avec d'autres variétés de riz introduites à cycle court comme les BG 90.2 et Dj 12119.

de riz Oryza sativa L Cultivar I PONK Pao (IPK) sélectionné in vitro en présence de sel, thèse, $140 \mathrm{p}$.

Durand J.H., Les sols irrigables. Agence de coopération culturelle et technique-conseil international de la langue française, 1983 Paris, 339p.

Diangar S., 2006. Approche intégrée pour l'amélioration de la productivité des systèmes de cultures à base de mil dans le bassin arachidier du Sénégal. Thèse de doctorat. Faculté des Sciences et Techniques/Université Cheikh Anta Diop de Dakar, $152 \mathrm{p}$.

Diop A., 1998. Salinité des sols de la moyenne vallée du fleuve du Sénégal: Zone de Mboro, aspects morphologiques et géochimiques. Mémoire Ise $77 \mathrm{p}$.

ISRA., 2012. Catalogue officiel des espèces et des variétés cultivées au Sénégal, Dakar, Sénégal, pp 60-67.

FAO., 2012. Adapter l'agriculture au changement climatique, 20p. 
Fall.A.C.A.L., 2002. Caractérisation des états de surface des sols sulfates acides salés sous aménagements biomécaniques dans le bassin fluvio-marin du Saloum, centre ouest du Sénégal : cas de Ndiafatte. Mémoire Ise, 71p.

Larcharme M., 2001.Quelques principes d'aménagement des rizières. $25 p$

Marius C., 1985. Mangrove du senegal et de la Gambie : écologie, pédologie, géochimie, mise en valeur et aménagement. Thèse doctorat, Université Louis Pasteur de Strasbourg, 335p.

Massibot, J., Carles, I., 1946. Mise en valeur des tannes rizicultivables du Sine (Sénégal).AGR, Nogent-Sur-Marne, Vol 1. 451p

Montoroi J.P., 1993. Les sols et l'agriculture dans le domaine estuarien de basse Casamance, 52$59 p$.

Ndiaye, A., Faye, E.H., Toure, M. A., 2014. Effets du stress salin sur la germination des graines de Gossypium hirsutum L. Journal of Applied Biosciences 80:7081 - 7092.12p

Ndiaye A., 2013. L'agriculture sénégalaise de 1958 à 2012; analyse systémique et prospective. Livre 224p.

Sow M.M., 1996. Etude du comportement de deux espèces de riz (Oryza sativa et Oryza glaberrima), mémoire ENSA, 125p.

Sadio S., 1991. Pédogenèse et potentialités forestières des sols sulfatés acides salés des tannes du sine Saloum (senegal), Thèse d'état, Orstom éditions, Dakar, 269p.

Sène J. H., Matty F., Diatta M., 2014. Caractérisation des sols de la vallée rizicole de Tamra dans l'île de Mar, Centre-Ouest du Sénégal. International journal biological and chemical sciences 8(2): 794-810,17p. 